Artículo científico

Volumen 30(3):803-819. Septiembre-diciembre, 2019

e-ISSN 2215-3608, doi:10.15517/am.v30i3.35645

http://www.revistas.ucr.ac.cr/index.php/agromeso

\title{
Evaluación de estratos arbóreos y arbustivos en un sistema silvopastoril en el trópico altoandino colombiano ${ }^{1}$
}

\section{Evaluation of arboreal and shrub strata in a silvopastoral system in Colombian high Andean tropics}

\author{
Laura Dayana Escobar-Pachajoa², Carolina Guatusmal-Gelpud ${ }^{3}$, Diego Hernán Meneses-Buitrago ${ }^{4}$, \\ Juan Leonardo Cardona-Iglesias ${ }^{4}$,Edwin Castro-Rincón ${ }^{4}$
}

1 Recepción: 9 de enero, 2019. Aceptación: 12 de marzo, 2019. Este trabajo formó parte del proyecto Mejoramiento de la oferta forrajera, optimización de sistemas de alimentación y aseguramiento de la calidad e inocuidad de leche en el Trópico Alto del Departamento de Nariño, desarrollado en la Corporación Colombiana de Investigación Agropecuaria (Agrosavia). Colombia.

2 Corporación Colombiana de Investigación Agropecuaria (AGROSAVIA) (hasta 21/08/2018). Nariño, Colombia. lauraescp@gmail.com

3 Corporación Colombiana de Investigación Agropecuaria (AGROSAVIA) (hasta 04/07/2018). Nariño, Colombia. carolinagg080@ gmail.com

4 Corporación Colombiana de Investigación Agropecuaria (AGROSAVIA). Nariño, Colombia. dmeneses@agrosavia.co (https://orcid.org/00000003-3033-3079), jlcardona@agrosavia.co, ecastro@agrosavia.co (autor para correspondencia; https://orcid.org/0000-0001-9841-8242).

\section{Resumen}

Introducción. Los sistemas silvopastoriles se presentan como una alternativa para el uso sostenible de los recursos naturales, por ello, surge la necesidad de identificar especies potenciales para su establecimiento en el trópico de altura. Objetivo. El objetivo fue evaluar el crecimiento inicial de ocho especies leñosas en un diseño silvopastoril para protección de fuente hídrica. Materiales y métodos. El estudio se realizó en el centro de investigación Obonuco de la Corporación Colombiana de Investigación Agropecuaria (Pasto, Colombia) entre septiembre 2017 y junio 2018 , se desarrolló un diseño de bloques al azar en arreglo factorial, donde interactuaron especies arbóreas/arbustivas diferenciadas por su hábito de crecimiento (estrato alto: Cedrela montana, Quercus humboldtii, Ficus andicola; estrato medio: Salix babylonica, Alnus acuminata, Smallanthus pyramidalis; estrato bajo: Morella pubescens y Sambucus nigra) y altitud (2750, 2770 y 2800), con cuatro repeticiones para evaluar variables morfométricas y calidad de plántulas. Resultados. En la variable altura, el mejor comportamiento se presentó en Ficus andicola a $2750 \mathrm{msnm}$ con 191,37 cm y Alnus acuminata a $2800 \mathrm{msnm}$ con 181,83 cm. En diámetro de tallo sobresalieron Ficus andicola a $2750 \mathrm{msnm}$ con 42,98 mm, Smallanthus pyramidalis a $2800 \mathrm{msnm}$ con 37,24 mm y Sambucus nigra a $2800 \mathrm{msnm}$ con $8,16 \mathrm{~mm}$. El número de rebrotes no evidenció efecto significativo de la interacción y, en calidad de plántulas, Quercus humboldtii a 2750 y 2700 msnm y Ficus andicola a 2750 msnm, presentaron la mejor calidad de individuos; mientras que, Cedrela montana mostró mortalidad en las tres altitudes evaluadas. Conclusiones. Las especies nativas sobresalieron por su capacidad de adaptación, reflejada en un rápido y óptimo crecimiento y desarrollo; lo cual evidencia su gran potencial para ser evaluadas en sistemas silvopastoriles de manera asociada, para determinar sus beneficios y potencial contribución con el desarrollo sostenible de la ganadería en la región.

Palabras clave: crecimiento, condición de la planta, cerca viva, sostenibilidad. 


\begin{abstract}
Introduction. Silvopastoral systems are presented as an alternative for the sustainable use of natural resources, so there is a need to identify potential species for their establishment in the high tropics. Objective. The objective was to evaluate the initial growth of eight woody species in a silvopastoral design for water source protection. Materials and methods. The study was carried out in the Obonuco research center of Corporacion Colombiana de Investigacion Agropecuaria (Pasto, Colombia) between September 2017 and June 2018. A randomized block design was developed in a factorial arrangement, where tree/bush species differentiated by their growth habit interacted (high stratum: Cedrela montana, Quercus humboldtii, Ficus andicola; middle stratum: Salix babylonica, Alnus acuminata, Smallanthus pyramidalis; lower stratum: Morella pubescens and Sambucus nigra) and altitude (2750, 2770 and 2800 masl) with four repetitions to evaluate morphometric variables and seedling quality. Results. In the variable height the best behavior was presented in Ficus andicola at 2750 masl with $191.37 \mathrm{~cm}$ and Alnus acuminata at 2800 masl with $181.83 \mathrm{~cm}$. In stem diameter Ficus andicola stood out at 2750 masl with $42.98 \mathrm{~mm}$, Smallanthus pyramidalis at 2800 masl with $37.24 \mathrm{~mm}$, and Sambucus nigra at 2800 masl with $8.16 \mathrm{~mm}$. The number of regrowths did not show a significant effect of the interaction, and as seedlings, Quercus humboldtii at 2750 and 2700 masl and Ficus andicola at 2750 masl showed the best quality of individuals; while Cedrela montana showed mortality at the three altitudes evaluated. Conclusions. Native species stood out for their adaptibility, reflected in fast and optimal growth and development, which evidences its great potential to be evaluated in silvopastoral systems in an associated way to decide its multiple benefits and potential contribution to the sustainable development of the livestock in the region.
\end{abstract}

Keywords: growth, plant condition, hedges, sustainability.

\title{
Introducción
}

La ganadería colombiana es una actividad productiva que contribuye al crecimiento económico, al desarrollo y a la satisfacción continua de la demanda de alimento en la población (Mahecha, 2004). En el departamento de Nariño, Colombia, la tierra se destina principalmente a la actividad pecuaria, dentro de la cual, la ganadería tiene gran relevancia, ya que el subsector lácteo, localizado principalmente en el trópico alto del departamento, es uno de los cinco núcleos más productivos del país (DNP, 2014). Sin embargo, uno de los mayores inconvenientes de la ganadería de ladera en la región es la afección de las fuentes superficiales de agua, originado por la tala de bosques y vegetación de las riberas, que genera una problemática ambiental (Libreros, 2015; UMAIC, 2017). Ante este contexto, surge la necesidad de una reconversión ganadera orientada hacia la producción sostenible, en este sentido, los sistemas silvopastoriles (SSP) se presentan como una alternativa que combina de forma simultánea árboles o arbustos con plantas herbáceas y animales. Estos sistemas de producción ofrecen beneficios como: la captura de carbono, protección de la biodiversidad, de los suelos y de las fuentes de agua; también son una alternativa forrajera para la alimentación y el bienestar animal, lo cual se asocia al mejoramiento de la calidad de los productos ganaderos (carne, leche, lana) (Montagnini, 2015; Murgueitio et al., 2015).

Los árboles dentro de las fincas pueden mejorar la conectividad entre los paisajes y, si se hallan de forma lineal, sirven de corredores biológicos para algunas especies, constituyéndose en áreas de amortiguamiento a lo largo de las praderas (DeFries y Rosenzweig, 2010). Por lo tanto, una de las estrategias planteadas para revertir la problemática y reducir el impacto de la ganadería sobre las corrientes de agua, es la implementación de corredores ribereños o cercas vivas, que son franjas de vegetación natural o introducidas, las cuales se dejan crecer a ambos lados de los cauces de ríos y quebradas (Uribe y Zuluaga, 2011). 
A pesar de las ventajas que ofrece la integración de árboles/arbustos mediante sistemas silvopastoriles en la ganadería bovina, aún existen limitantes técnicas, entre las cuales se encuentran el establecimiento de germoplasma no adaptable a las zonas y el mal uso de los recursos arbóreos nativos (Clavero y Suárez, 2006; Calle et al., 2012; Zepeda et al., 2016). Es importante considerar que, con el fin de restaurar ecosistemas auto sostenibles y sus servicios, las especies nativas son generalmente preferidas sobre especies exóticas o introducidas, ya que, las especies nativas generalmente se adaptan mejor a las condiciones bióticas y abióticas locales, y de esta manera promueven la biodiversidad nativa y la función de los ecosistemas en mayor grado que las exóticas (Tang et al., 2007).

Para la región altoandina son pocas las investigaciones en cuanto al comportamiento adaptativo de leñosas perennes, que permitan identificar especies potenciales para la zona de vida. Debido a lo anterior, es necesario evaluar especies arbóreas y arbustivas, que al implementarse en áreas de producción ganadera posean equilibrio y adaptabilidad ambiental, que garanticen y prolonguen la vida útil del sistema (Flebes y Ruiz, 2008). Por ejemplo, la especie Morella pubescens se reconoce como nativa promisoria en altitudes entre 1600 y $3200 \mathrm{msnm}$, en donde crece de manera natural en los potreros, cerca de los ríos y quebradas, y destaca en sistemas silvopastoriles por la capacidad de captura de carbono; además, presenta un alto potencial económico e industrial, debido a la extracción de la cera de sus hojas (Luna, 2011; Delgado et al., 2016). De igual manera, Sambucus nigra es una especie que, de acuerdo con sus características, manejo, usos y beneficios, tiene un potencial para implementación en sistemas silvopastoriles del trópico alto (Grajales et al., 2015).

Es importante considerar que la altitud influye sobre la tasa de crecimiento y desarrollo de las plantas, ya que puede afectar la dinámica de los ecosistemas, mediante cambios en la fenología, fisiología o dinámica poblacional de las especies (Machado et al., 2006; Parmesan, 2006). En el presente estudio, por medio de estaciones meteorológicas automáticas ubicadas en el centro de investigación Obonuco, se identificó que en el rango de altitudes manejadas (2750-2700-2800 msnm), los valores de temperatura máxima variaron entre $18-20,5{ }^{\circ} \mathrm{C}$ y la mínima entre 4,7-8 ${ }^{\circ} \mathrm{C}$; lo que evidencia las diferencias marcadas de temperatura. En este contexto, el objetivo de esta investigación fue evaluar el crecimiento inicial de ocho especies leñosas en un sistema silvopastoril para protección de fuente hídrica.

\section{Materiales y métodos}

\section{Localización}

El experimento se llevó a cabo en el Centro de Investigación (C.I) Obonuco, perteneciente a la Corporación Colombiana de Investigación Agropecuaria (AGROSAVIA), ubicado en el municipio de Pasto, Colombia. El estudio se llevó a cabo entre septiembre del 2017 y junio del 2018 (nueve meses). En el período de investigación se tuvo una temperatura promedio de $12,8^{\circ} \mathrm{C}$, una precipitación promedio anual de $619,7 \mathrm{~mm}$, humedad relativa del $84,4 \%$ y la velocidad del viento varió entre 1,2 y $2,1 \mathrm{~m} \mathrm{~s}^{-1}$. Se evaluó un sistema silvopastoril cerca viva multiestrato protector de fuente hídrica, ubicado a $1^{\circ} 11^{\prime} 42^{\prime \prime} \mathrm{N} 77^{\circ} 18^{\prime} 38^{\prime \prime} \mathrm{W}$ en una zona de vida Bosque seco Montano bajo (BsMb) a 2750-2800 msnm, según los parámetros de Holdridge (2000).

El cerco vivo multiestrato estuvo compuesto por ocho especies arbóreas y arbustivas (Cuadro 1), que se establecieron a lo largo del borde de un canal de agua de $960 \mathrm{~m}$ de longitud.

\section{Variables evaluadas}

Las mediciones se desarrollaron en diferentes estratos arbóreos/arbustivos (alto, medio y bajo) cada tres meses. Para identificar el crecimiento inicial de cada especie, se tomaron las variables morfométricas y de la calidad de 
Cuadro 1. Estructura, composición y función del diseño silvopastoril cerco vivo multiestrato, establecido en el Centro de Investigación Obonuco, Corporación Colombiana de Investigación Agropecuaria. Pasto, Colombia. 2018.

Table 1. Structure, composition, and function of the design of a live multi-layer protective fence, established in the Obonuco Research Center, Corporación Colombiana de Investigación Agropecuaria. Pasto, Colombia. 2018.

\begin{tabular}{lcccc}
\hline Nombre común & Nombre científico & Estrato & Distancia siembra (m) & Usos \\
\hline Cedro & Cedrela montana Moritz ex Turcz & Alto & $40 * 40$ & MF, LÑ , \\
Caucho sabanero & Ficus andicola Standl & Alto & $40 * 40$ & PT \\
Roble andino & Quercus humboldtii Bonpl & Alto & $40 * 40$ & MF, LN \\
Aliso & Alnus acuminata Kunth & Medio & $20 * 20$ & MF,LÑ , FR \\
Sauce & Salix babylonica L & Medio & $20 * 20$ & MF,LN \\
Árboloco & Smallanthus pyramidalis (Triana) H. Rob & Medio & $20 * 20$ & PT \\
Laurel de cera & Morella pubescens (Humb. \& Bonpl.) & Bajo & $2 * 2$ & PT \\
Sauco & Wilbur & Bajo & $2 * 2$ & FR \\
\hline
\end{tabular}

MF: madera fina, LÑ: leña, FR: forraje, PT: protección de fuentes hídricas y biodiversidad / MF: fine wood, LÑ: wood, FR: forage, P: protection of water sources and biodiversity.

plantas, de acuerdo con la metodología de Sánchez y Murillo (2004), la cual integra altura (cm) (por medio de una regla graduada), diámetro basal $(\mathrm{mm})$ (con un paquímetro digital $150 \mathrm{~mm}$ ), número de rebrotes (conteo visual), características como presencia de bifurcaciones y malezas (0:ausencia, 1:presencia), daño mecánico (0:sin daño, 1 :con daño), meristemo apical ( 0 : sano, 1: dañado), rectitud del tallo ( 0 : recto, 1 : con torceduras severas anormales en la especie) y estado fitosanitario (sanidad asociada a plagas y enfermedades 1: plántula completamente sana sin ningún problema fitosanitario visible, de buen color y vigor, 2: plántula relativamente sana con alguna evidencia de problemas fitosanitarios, pero que no corre riesgo de morir y principalmente cuando no se presente en más de un $50 \%$ del follaje o que no haya provocado heridas severas en el tallo y 3: plántula enferma, con problemas fitosanitarios que afectan el desarrollo normal de la plántula tales como la pérdida del eje dominante o del follaje y en general, daños visibles en más del $50 \%$ de la planta). Con base en las características anteriores, se determinó la calidad de plántulas en escala de 1 a 3 en donde 1: plántula con ausencia absoluta de características indeseables (daño mecánico, malezas, torceduras y enfermedades), 2: plántula de sanidad 2 o con calificación de 1 en alguna de las características anteriores y 3: plántula de mala calidad asociada a sanidad 3 o mortalidad.

\section{Diseño experimental y análisis estadístico}

Se utilizó un diseño de bloques al azar con arreglo factorial. Se realizaron análisis de varianza, en los que se controlaron los efectos de especies arbóreas y arbustivas (8), la altitud (3) y su interacción (8 x 3), con un total de veinticuatro tratamientos con cuatro repeticiones (Cuadro 2). Para el análisis de los datos se agruparon las especies, donde para cada grupo se estudió la interacción con los tres niveles de altitud (2750, 2770 y 2800 msmn).

Para los datos morfométricos se emplearon variables cuantitativas (altura, diámetro basal, número de rebrotes) y variables cualitativas (calidad de plántula). Posteriormente, los datos se analizaron dependiendo de su varianza y distribución normal, para lo cual se realizaron análisis de varianza acompañados de la prueba de comparación de medias de Tukey $(\alpha=0,05)$ para las variables altura (porte medio y bajo) y diámetro (porte bajo), y la prueba de Friedman acompañada de una comparación de medianas LSD $(\alpha=0,05)$ para las variables altura (porte medio), diámetro (porte alto y medio) y número de rebrotes (porte alto, medio y bajo). La variable calidad se analizó a 
Cuadro 2. Tratamientos para evaluar el crecimiento y adaptación de ocho especies arbóreas/arbustivas en un diseño silvopastoril cerco vivo multiestrato, establecido como protector de fuente hídrica en el Centro de Investigación Obonuco, Corporación Colombiana de Investigación Agropecuaria (Agrosavia), Pasto, Colombia. 2018.

Table 2. Treatments to evaluate the growth and adaptation of eight tree/shrub species in a multi-layer live fence silvopastoral design, established as water source protector in the Research Center Obonuco, Corporación Colombiana de Investigación Agropecuaria (Agrosavia), Pasto, Colombia. 2018.

\begin{tabular}{cccccc}
\hline Trat. & Altitud $(\mathbf{m s n m})$ & Especie & Trat. & Altitud $(\mathbf{m s n m})$ & Especie \\
\hline $\mathbf{1}$ & 2800 & Cedrela montana & 13 & 2770 & Salix babylonica \\
$\mathbf{2}$ & 2800 & Quercus humboldtii & 14 & 2770 & Alnus acuminata \\
$\mathbf{3}$ & 2800 & Ficus andicola & 15 & 2770 & Smallanthus pyramidalis \\
$\mathbf{4}$ & 2770 & Cedrela montana & 16 & 2750 & Salix babylonica \\
$\mathbf{5}$ & 2770 & Quercus humboldtii & 17 & 2750 & Alnus acuminata \\
$\mathbf{6}$ & 2770 & Ficus andicola & 18 & 2750 & Smallanthus pyramidalis \\
$\mathbf{7}$ & 2750 & Cedrela montana & 19 & 2800 & Morella pubescens \\
$\mathbf{8}$ & 2750 & Quercus humboldtii & 20 & 2800 & Sambucus nigra \\
$\mathbf{9}$ & 2750 & Ficus andicola & 21 & 2770 & Morella pubescens \\
$\mathbf{1 0}$ & 2800 & Salix babylonica & 22 & 2770 & Sambucus nigra \\
$\mathbf{1 1}$ & 2800 & Alnus acuminata & 23 & 2750 & Morella pubescens \\
$\mathbf{1 2}$ & 2800 & Smallanthus pyramidalis & 24 & 2750 & Sambucus nigra \\
\hline
\end{tabular}

través de tablas de contingencia, acompañadas del estadístico de chi-cuadrado de Pearson $(\alpha=0,05)$. Los cálculos se hicieron con base en el software estadístico R. V.3.5.1. Se emplearon los paquetes agricolae (Mendiburu, 2017) y ggplot2 (Wickham, 2016) para el análisis.

\section{Resultados}

\section{Altura}

En la variable altura hubo diferencias significativas $(\mathrm{p}<0,05)$, donde $F$. andicola a $2750 \mathrm{msnm}$ obtuvo el mayor valor con una media de $191,31 \mathrm{~cm}$ y diámetro de tallo de $42,98 \mathrm{~mm}$; mientras que C. montana a $2750 \mathrm{msnm}$ registró la mínima altura $(39 \mathrm{~cm})$ y diámetro $(9,5 \mathrm{~cm})$ (Figura 1).

En el estrato medio el mejor comportamiento en altura se presentó en las especies A. acuminata y $S$.pyramidalis a $2800 \mathrm{msnm}$, con medianas de $181,83 \mathrm{~cm}$ y $167,66 \mathrm{~cm}$, respectivamente (Figura 2).

En el estrato bajo no se presentaron diferencias significativas. Sin embargo, se evidenció que M. pubescens a $2800 \mathrm{msnm}$ presentó una tendencia superior al resto de los tratamientos con una media de 70,80 cm (Figura 3).

\section{Diámetro basal}

En la variable diámetro basal se encontraron diferencias significativas en el estrato alto y medio, se observó que entre las especies arbóreas de porte alto $F$. andicola a $2750 \mathrm{msnm}$, presentó la mayor mediana con 42,98 mm (Figura 4). En el estrato medio sobresalió la especie S. pyramidalis a $2800 \mathrm{msnm}$ con $37,24 \mathrm{~mm}$ de diámetro (Figura 5). 


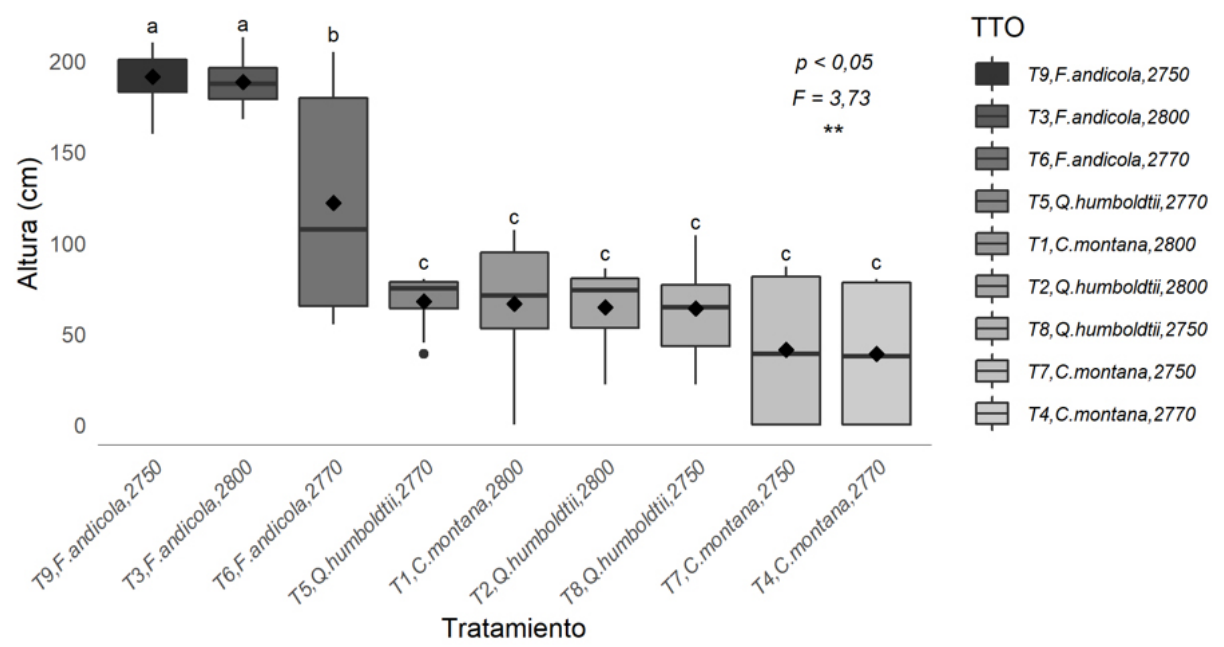

a,b,c,d medias con letras diferentes en las barras difieren entre si según la prueba de Tukey $(\mathrm{p}<0,05)$. ** Probabilidades por debajo de $5 \%$ representan diferencias altamente significativas. Representa la media aritmética / a,b,c,d averages with different letters on the bars differ from each other according to Tukey's test $(\mathrm{p}<0.05)$. ** Probabilities below $5 \%$ represent highly significant differences. Represents the arithmetic mean.

Figura 1. Efecto de la altitud sobre la altura en especies arbóreas de estrato alto, en el Centro de Investigación Obonuco, Corporación Colombiana de Investigación Agropecuaria. Pasto, Colombia. 2018.

Figure 1. Effect of altitude on height in high stratum tree species, in the Obonuco Research Center, Corporación Colombiana de Investigación Agropecuaria. Pasto, Colombia. 2018.

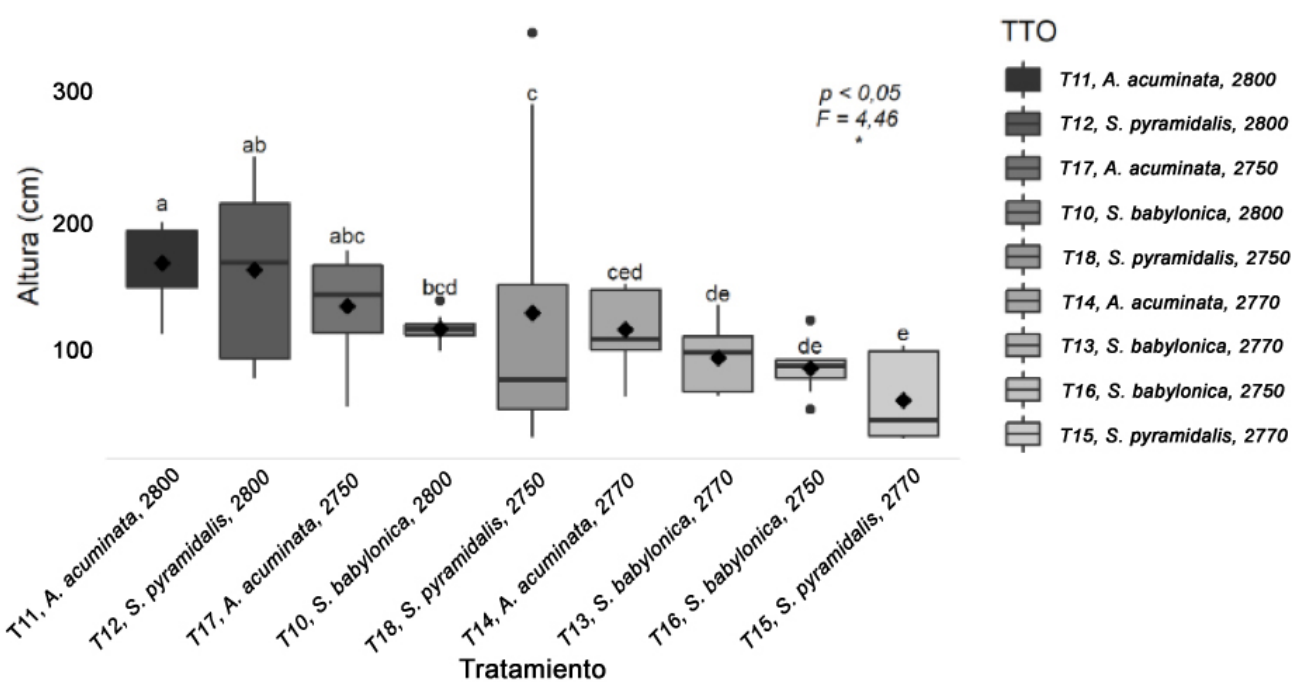

a,b,c,d medianas con letras diferentes en las barras difieren entre si según la prueba de Friedman $(\mathrm{p}<0,05) . *$ Probabilidades representan diferencias significativas. Representa la media aritmética / a,b,c,d medians with different letters on the bars differ from each other according to the Friedman test $(\mathrm{p}<0.05)$. * Probabilities represent significant differences. $\bullet$ Represents the arithmetic mean.

Figura 2. Efecto de la altitud sobre la altura en especies arbóreas de estrato medio, en el Centro de Investigación Obonuco, Corporación Colombiana de Investigación Agropecuaria. Pasto, Colombia. 2018.

Figure 2. Effect of altitude on height in middle stratum tree species, in the Obonuco Research Center, Corporación Colombiana de Investigación Agropecuaria. Pasto, Colombia. 2018. 


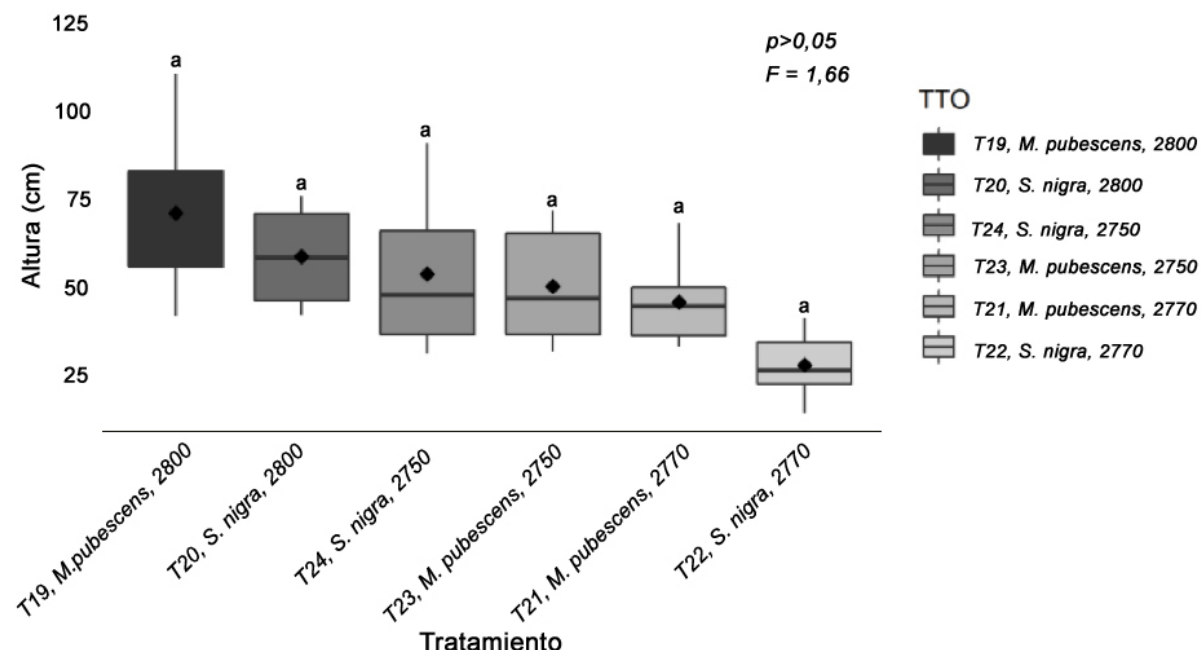

a,b,c,d medias con letras diferentes en las barras difieren entre si según la prueba de Tukey $(\mathrm{p}<0,05)$. Representa la media aritmética / $\mathrm{a}, \mathrm{b}, \mathrm{c}, \mathrm{d}$ averages with different letters on the bars differ from each other according to Tukey's test $(\mathrm{p}<0.05)$. $\diamond$ Represents the arithmetic mean.

Figura 3. Efecto de la altitud sobre la altura en especies arbóreas de estrato bajo en el Centro de Investigación Obonuco, Corporación Colombiana de Investigación Agropecuaria. Pasto, Colombia. 2018.

Figure 3. Effect of altitude on height in low stratum tree species in the Obonuco Research Center, Corporación Colombiana de Investigación Agropecuaria. Pasto, Colombia. 2018.

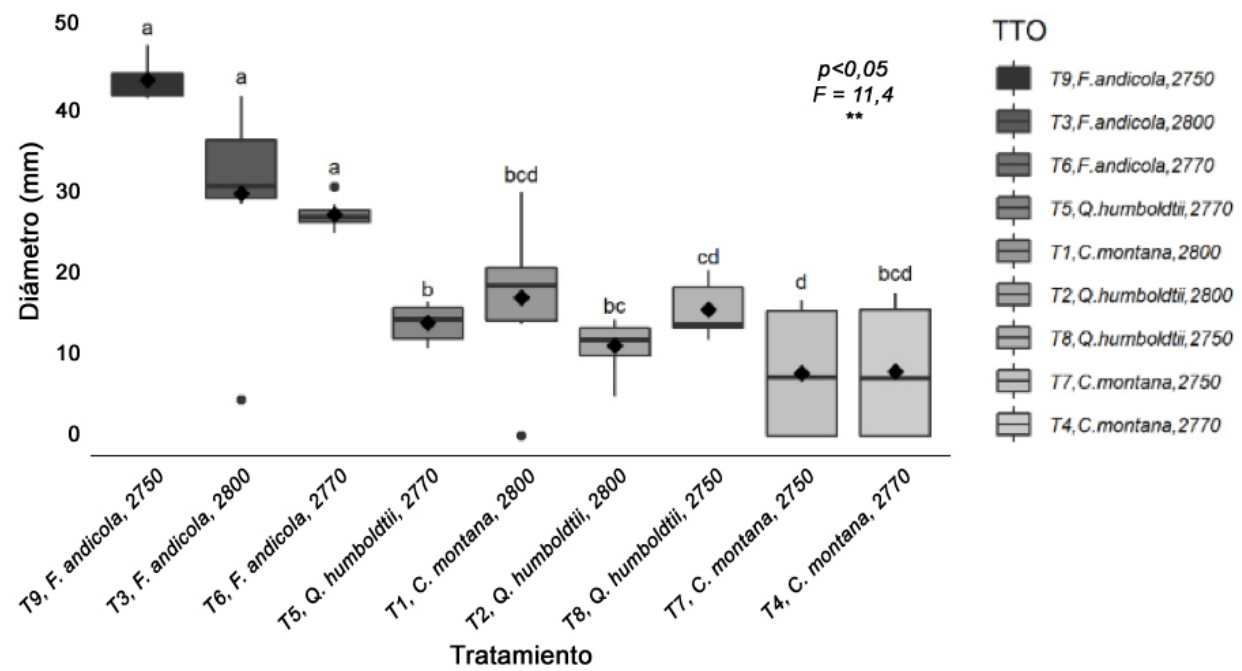

a,b,c,d medianas con letras diferentes en las columnas difieren entre si según la prueba de Friedman $(\mathrm{p}<0,05)$. ** Probabilidades por debajo de 5\% representan diferencias altamente significativas. Representa la media aritmética / a,b,c,d medians with different letters in the columns differ from each other according to Friedman's test $(\mathrm{p}<0.05)$. ** Probabilities below 5\% represent highly significant differences. Represents the arithmetic mean.

Figura 4. Efecto de la altitud sobre el diámetro en especies arbóreas de estrato alto en el Centro de Investigación Obonuco, Corporación Colombiana de Investigación Agropecuaria, Pasto, Colombia. 2018.

Figure 4. Effect of altitude on diameter in upper stratum tree species at the Obonuco Research Center, Corporación Colombiana de Investigación Agropecuaria, Pasto, Colombia. 2018. 


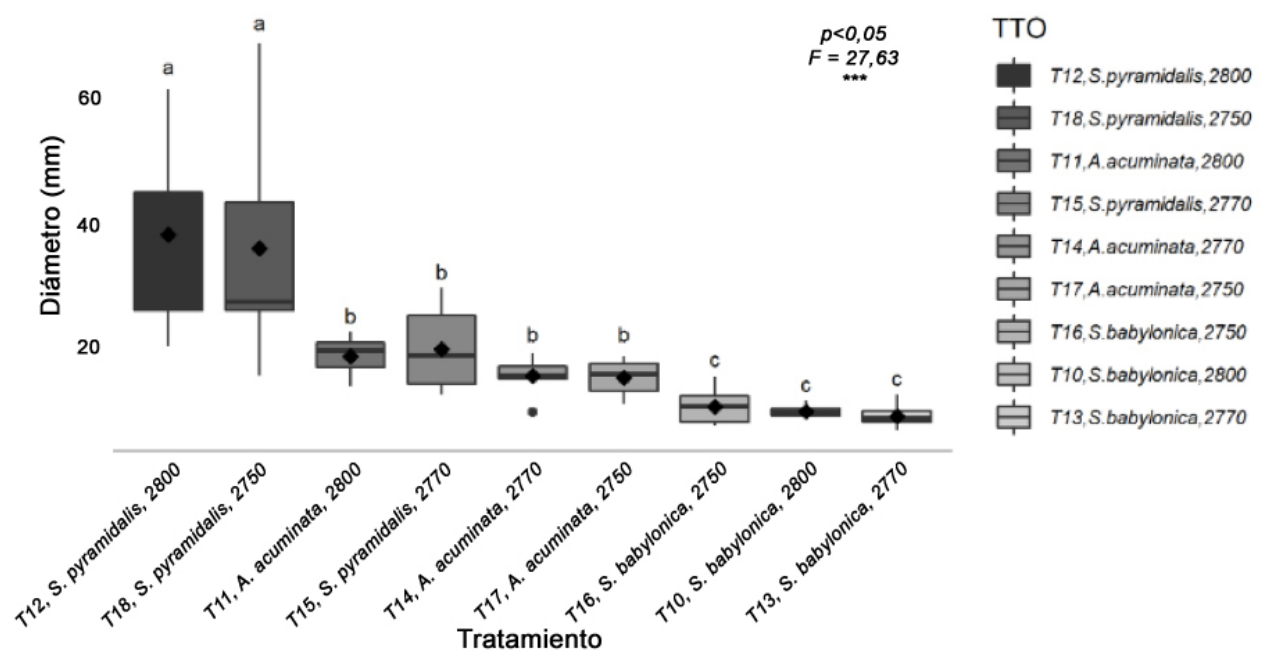

a,b,c,d medianas con letras diferentes en las columnas difieren entre si según la prueba de Friedman ( $<<0,05)$. *** Probabilidades por debajo de 5\% representan diferencias extremadamente significativas. Representa la media aritmética / a,b,c,d medians with different letters in the columns differ from each other according to the Friedman test $(\mathrm{p}<0.05)$. *** Probabilities below 5\% represent extremely significant differences. Represents the arithmetic mean.

Figura 5. Efecto de la altitud sobre el diámetro en especies arbóreas de estrato medio en el Centro de Investigación Obonuco, Corporación Colombiana de Investigación Agropecuaria. Pasto, Colombia. 2018.

Figure 5. Effect of altitude on diameter in middle stratum tree species in the Obonuco Research Center, Corporación Colombiana de Investigación Agropecuaria. Pasto, Colombia. 2018.

El análisis de varianza, para el estrato bajo en la variable diámetro no mostró diferencias $(\mathrm{p}>0,05)$ en los tratamientos. Sin embargo, se evidenció que la especie $S$. nigra a 2800 y 2750, presentó un diámetro superior a $M$. pubencens (Figura 6).

\section{Número de rebrotes}

Los resultados no mostraron diferencias significativas para la variable número de rebrotes $(p \geq 0,05)$ en los tres estratos evaluados (Figuras 7, 8 y 9).

\section{Calidad de plantas}

$\mathrm{Al}$ analizar la calidad de plantas, se encontraron diferencias en el estrato alto, donde $Q$. humboldtii a 2770 y $2750 \mathrm{msnm}$ y $F$. andicola a $2750 \mathrm{msnm}$, presentaron la mejor calidad de plantas (1). Mientras que la especie $C$. montana a 2770 y $2750 \mathrm{msnm}$, mostró un alto porcentaje de mortalidad.

En el estrato medio y bajo, la calidad de la plántula no parece haber sido influenciada por los tratamientos, ya que se observó una calidad que varió entre 1 y 2 , es decir, para estos estratos no se reportó mortalidad de las especies. No obstante, en el estrato medio sobresalieron. S. babylonica a $2800 \mathrm{msnm}$, S. pyramidalis a $2800 \mathrm{msnm}$ y S. babylonica a $2770 \mathrm{msnm}$, con calidad 1 en más del $70 \%$ de los individuos; mientras que A. acuminata a 2770 msnm y 2750 msnm presentó mayor afectación (calidad 2). En el estrato bajo sobresalió M. pubescens a 2800 msnm, por presentar calidad 1 en el $100 \%$ de individuos evaluados. 


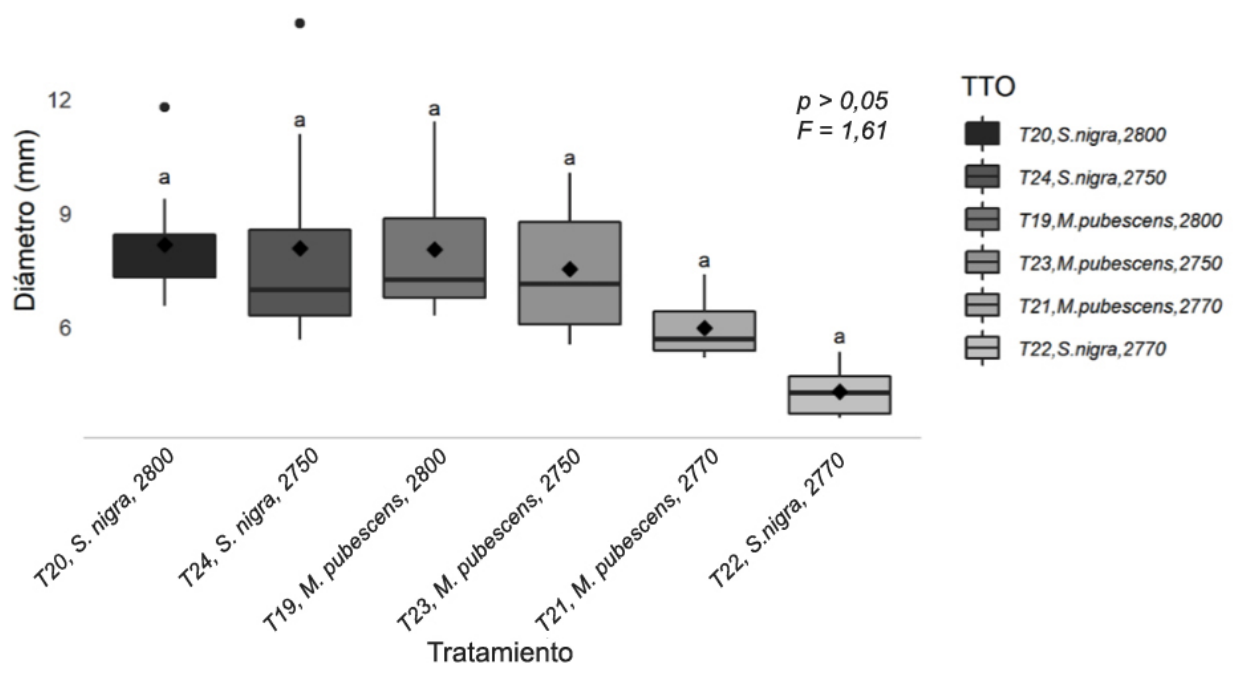

a,b,c,d medias con letras diferentes en las columnas difieren entre si según la prueba de Tukey ( $<<0,05)$. $\bullet$ Representa la media aritmética / a,b,c,d averages with different letters in the columns differ from each other according to Tukey's test $(\mathrm{p}<0.05)$. Represents the arithmetic mean.

Figura 6. Efecto de la altitud sobre el diámetro en especies arbóreas de estrato bajo en el Centro de Investigación Obonuco, Corporación Colombiana de Investigación Agropecuaria. Pasto, Colombia. 2018.

Figure 6. Effect of altitude on diameter in low stratum tree species in the Obonuco Research Center, Corporación Colombiana de Investigación Agropecuaria. Pasto, Colombia. 2018.

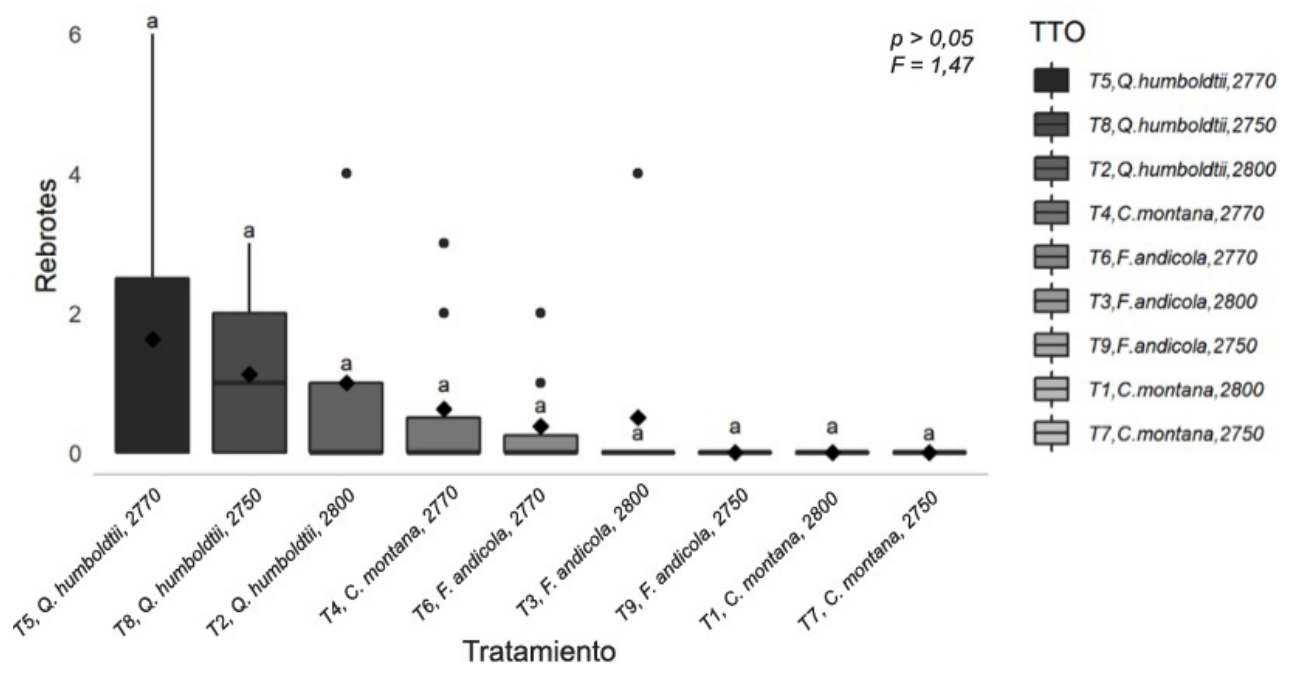

a,b,c medianas con letras diferentes en las barras difieren entre sí, según la prueba de Friedman $(\mathrm{p}<0,05)$. Representa la media aritmética / a,b,c medians with different letters on the bars differ from each other, according to the Friedman test $(\mathrm{p}<0.05)$. Represents the arithmetic mean.

Figura 7. Efecto de la altitud sobre el número de rebrotes en especies arbóreas de estrato alto en el Centro de Investigación Obonuco, Corporación Colombiana de Investigación Agropecuaria. Pasto, Colombia. 2018.

Figure 7. Effect of altitude on the number of regrowths in tree species of high stratum in the Obonuco Research Center, Corporación Colombiana de Investigación Agropecuaria. Pasto, Colombia. 2018. 
5

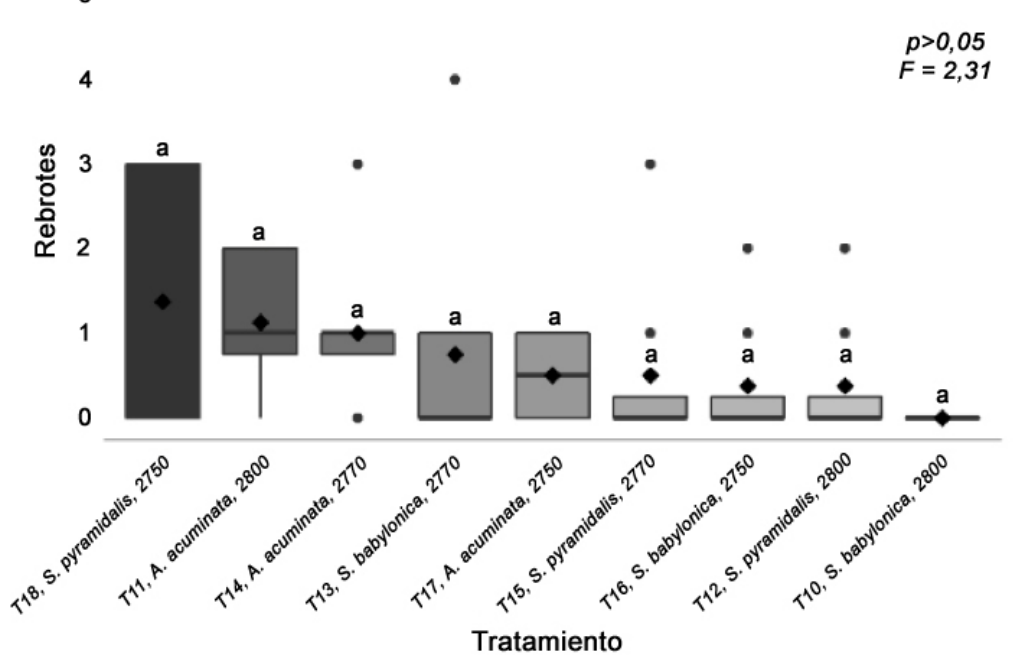

TTO

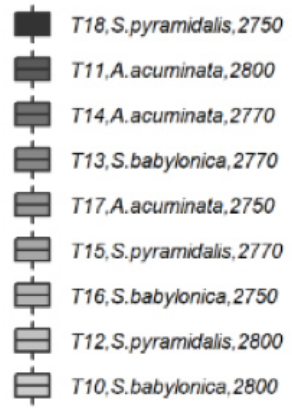

a,b,c medianas con letras diferentes en las barras difieren entre sí, según la prueba de Friedman $(\mathrm{p}<0,05)$. Representa la media aritmética / a,b,c medians with different letters on the bars differ from each other, according to the Friedman test $(\mathrm{p}<0.05)$. $\downarrow$ Represents the arithmetic mean.

Figura 8. Efecto de la altitud sobre el número de rebrotes en especies arbóreas de estrato medio en el Centro de Investigación Obonuco, Corporación Colombiana de Investigación Agropecuaria, Colombia. 2018.

Figure 8. Effect of altitude on the number of regrowths in middle-stratum tree species at the Obonuco Research Center, Corporación Colombiana de Investigación Agropecuaria, Colombia. 2018.

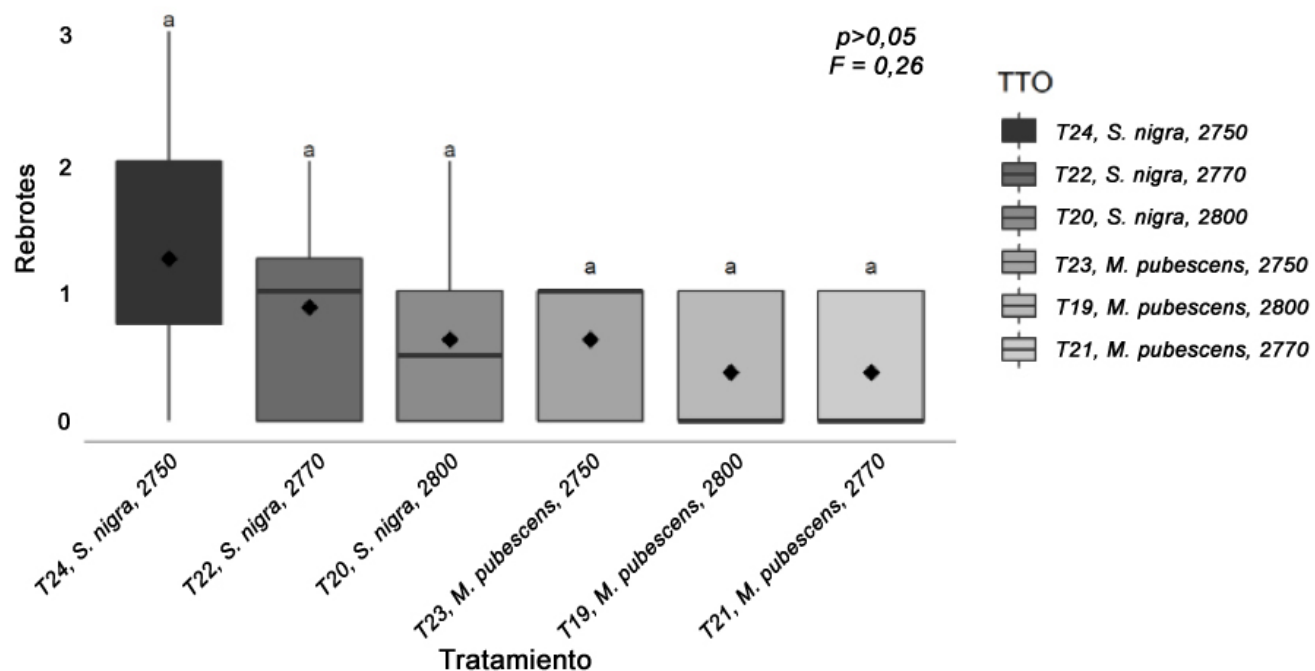

a,b,c medianas con letras diferentes en las barras difieren entre sí, según la prueba de Friedman $(\mathrm{p}<0,05)$. Representa la media aritmética / a,b,c medians with different letters on the bars differ from each other, according to the Friedman test ( $\mathrm{p}<0.05)$. $\bullet$ Represents the arithmetic mean.

Figura 9. Efecto de la altitud sobre el número de rebrotes en especies arbóreas de estrato bajo en el Centro de Investigación Obonuco, Corporación Colombiana de Investigación Agropecuaria, Pasto, Colombia. 2018.

Figure 9. Effect of altitude on the number of regrowths in low stratum tree species in the Obonuco Research Center, Corporación Colombiana de Investigación Agropecuaria, Pasto, Colombia. 2018. 


\section{Discusión}

En la investigación se identificó que la altitud tuvo influencia sobre el crecimiento de las especies; en el estrato alto se destacó $F$. andicola, ya que registró la mayor altura y diámetro de tallo a una altitud de $2750 \mathrm{msnm}$, lo cual es un resultado esperado, debido a que se reconoce como una especie nativa de Colombia que crece entre 1800 y 3000 msnm (Estupiñán y Cabrera, 2008; Tovar, 2013). Varios autores han reportado la presencia de la especie en este rango, Cardona et al. (2007) registraron que $F$. andicola crece en forma natural en el bosque primario en el municipio de Pereira (Risaralda-Colombia) de 1800 a 2100 msnm; Sierra y Amarillo (2017) señalaron el crecimiento espontáneo y óptimo de la especie en varias localidades de Bogotá; además, Núñez-Florez et al. (2019) resaltaron la importancia de la implementación de esta especie en Bogotá a una altitud de 2560 msnm, con el fin de conservar la biodiversidad y brindar sombra. Estos reportes y los resultados obtenidos en este estudio, permiten afirmar que la especie tiene un rango altitudinal amplio, se distribuye principalmente en la zona central de Colombia. En Nariño, no se encontraron estudios que indiquen la presencia de la especie, sin embargo, de acuerdo con los resultados se demuestra la capacidad de adaptación de esta a las condiciones de la región.

En el estrato alto, la especie $C$. montana presentó un bajo crecimiento en altura y diámetro, resultados similares a los reportados por González et al. (2010), quienes encontraron un bajo crecimiento de esta especie en las altitudes evaluadas (1850 a $2220 \mathrm{msnm}$ ). Los mismos autores explicaron este comportamiento mediante la muerte lenta de tallos, que rebrotaron nuevamente a partir de la base difiriendo su altura, lo cual concuerda con la presente investigación, ya que se observó en algunas plántulas la pérdida de las hojas y desecación de la parte apical del tallo; no obstante, en la base se generaron rebrotes, por lo cual la medición de altura varió. El desmoche (recorte indiscriminado de las ramas dejando muñones o ramas laterales), no permite a las nuevas ramas asumir el papel terminal, causado por un daño mecánico o por muerte parcial de la parte apical del tallo (Rivas, 2005).

En el estrato arbóreo de porte medio, A. acuminata y S. pyramidalis a $2800 \mathrm{msnm}$ presentaron los mayores valores de altura. Estas especies son reconocidas ecológicamente como pioneras en los estadios de sucesión del bosque altoandino, asociadas a un rápido crecimiento (Pacheco y Quisbert, 2016). El desarrollo de A. acuminata, generalmente está restringido a zonas con humedad del suelo, como las tierras altas tropicales y frías (Orwa et al., 2009). Según lo anterior, se podría inferir que el comportamiento presentado en este estudio puede relacionarse con la disponibilidad de recurso hídrico permanente que proveía la presencia de una quebrada, a lo largo de la cual se establecieron los árboles de A. acuminata.

Al observar el comportamiento en altura de A. acuminata a $2950 \mathrm{msnm}$ y $3150 \mathrm{msnm}$, Sánchez et al. (2010) afirmaron que, a mayor altitud y menor precipitación, el crecimiento de los árboles de esta especie fue menor; valores asociados con lo reportado por dichos autores se observaron al comparar estudios desarrollados por Molina et al. (2008), quienes encontraron un incremento en altura de $0,30 \mathrm{~cm}$ día ${ }^{-1}$ en una localidad del municipio de Jardín (Antioquia, Colombia), a $2000 \mathrm{msnm}$ con precipitaciones entre 2000 y $4000 \mathrm{~mm}$ anuales. Se reportaron $0,22 \mathrm{~cm} \mathrm{día}^{-1}$ a $2475 \mathrm{msnm}$ con precipitación anual promedio de $1575 \mathrm{~mm}$ (Medina et al., 2008). De igual manera, Bare y Ashton (2015) observaron crecimiento de 0,26 cm día ${ }^{-1}$ en los Andes Tropicales, localizados entre 1000 a 3200-3500 msnm con precipitaciones de $700 \mathrm{~mm}$ y $1700 \mathrm{~mm}$ anuales. Sin embargo, en la presente investigación se observó un incremento en altura para A. acuminata de $0,43 \mathrm{~cm}_{\text {día }}{ }^{-1}$ a $2800 \mathrm{msnm}$ con precipitación de $619,7 \mathrm{~mm}$ anuales, evidenciando un mayor incremento de altura con menor precipitación en comparación con los reportes mencionados anteriormente.

S. pyramidalis también se destacó como una de las especies con mayor incremento de altura a $2800 \mathrm{msnm}$; esta especie se reconoce como un árbol adaptado al bosque andino bajo pionero en procesos de restauración ecológica, por lo que tiende a crecer rápidamente (Fernández y Hernández, 2007; García, 2009). Similar a los resultados de este estudio, León (2007) y Ávila y Vargas (2009), en investigaciones realizadas entre los 3000 y 3300 msnm, reportaron a $S$. pyramidalis como la especie con mayor crecimiento vertical. Lo anterior se relaciona con 
el crecimiento en diámetro que evidenció el mejor comportamiento en $S$. pyramidalis a 2800 y $2750 \mathrm{msnm}(0,013$ $\mathrm{cm}$ día ${ }^{-1}$ y $0,010 \mathrm{~cm} \mathrm{día}^{-1}$, respectivamente). Se encontraron en esta especie incrementos de $0,33 \mathrm{~cm} \mathrm{día}{ }^{-1}$ en altura y $0,004 \mathrm{~cm}$ día ${ }^{-1}$ en diámetro, y además, el bajo crecimiento en diámetro reportado por Bare y Ashton (2015), en comparación con la presente investigación, puede estar relacionado a que no delimitaron la zona de estudio, ya que incluyeron observaciones desde 1000 a 3200-3500 msnm. Dentro del estudio de Bare y Ashton (2015), se reportó menor adaptación de $S$. pyramidalis a mayores alturas; mientras que en el presente estudio se tomaron datos de tres elevaciones específicas, de las cuales 2800 msnm correspondiente a la máxima evaluada, presentó las mejores condiciones para el desarrollo diametral de esta especie; que de acuerdo con Apráez et al. (2014) es potencial dentro de sistemas silvopastoriles por la calidad nutricional de su forraje.

Al realizar una comparación entre las dos especies de porte bajo se pudo observar que $M$. pubescens superó a $S$. nigra en altura, pero $S$. nigra fue mayor en diámetro, estos resultados probablemente se presentaron debido a que $S$. nigra tiene un comportamiento de crecimiento de una especie arbustiva con una ramificación desde la base del tallo, su tronco es curvo e inclinado, con corteza rugosa y ramas gruesas (Sánchez et al., 2010). Por lo tanto, es normal que esta especie tenga un diámetro mayor y menor altura cuando se la compara con M. pubescens.

Se encontró una tasa de crecimiento de las plántulas de $S$. nigra en la Sabana de Bogotá a los nueve meses de 48,4 cm (Millán y Moreno, 2005), mientras que en la presente investigación registró una altura promedio de 58,54 $\mathrm{cm}$, lo cual indica que, aunque la especie no obtuvo la mayor altura en el estrato bajo, se adaptó adecuadamente a las condiciones en las cuales se trabajó. Esta especie se ha aclimatado en muchas regiones, incluso en las alturas andinas (Blanco et al., 2005), y es común encontrarla en el departamento de Nariño (Muñoz et al., 2018). Adicionalmente, esta especie se presenta como una alternativa de alimentación animal, razón por la cual es común encontrarla en sistemas silvopastoriles (Cárdenas et al., 2011), donde se le considera como una especie multipropósito, ya que, por sus características, permite ser implementada bajo diferentes formas de manejo, para proporcionar forraje de buena calidad al ganado, mejorar condiciones para aumentar la calidad de la leche, también aporta en la protección de los recursos (suelo-agua) y, en general, ofrece diversidad de subproductos que pueden ser aprovechados (Grajales et al., 2015). De acuerdo con lo anterior, S. nigra se presenta como una especie potencial para establecer en los sistemas silvopastoriles en el trópico alto de Nariño.

Para la especie S. pyramidalis se reportó que, generalmente, se adapta bien a los trópicos de altura en Colombia. En bosque altoandino del Parque Forestal Embalse del Neusa, Cundinamarca, se observó que $S$. pyramidalis fue una de las especies que más rebrotes registró y varió entre uno y seis (Gutiérrez, 2012). Dentro de la presente investigación, el máximo rebrote reportado fue uno, lo cual puede estar asociado a la buena calidad de las yemas terminales de la especie, relacionada con una dominancia apical que genera un efecto inhibidor de las partes apicales de la planta sobre las yemas laterales, debido a que en las yemas apicales normalmente se producen hormonas de crecimiento como las auxinas.

Con base en el potencial de rebrote encontrado en el presente estudio, se puede inferir que las especies $Q$. humboldtii, A. acuminata y S. nigra, pueden aportar a la sostenibilidad del sistema evaluado, debido a su mayor tendencia a sobrevivir mediante la formación de rebrotes, lo cual se puede asociar con una capacidad para almacenar en sus raíces grandes cantidades de sustancias de reserva para poder regenerar biomasa aérea (Pausas, 2010). En este contexto, estas especies pueden ser adecuadas para establecimiento de sistemas silvopastoriles en el trópico de altura, dentro de lo cual la capacidad de rebrote es fundamental, ya que se asocia al potencial de recuperación de las especies después de labores de poda y posibles daños mecánicos después de ramoneo directo.

La buena calidad de plantas observada en $Q$. humboldtii y $F$. andicola, puede estar relacionada con que estas especies se reportan como nativas, por tanto, responden con una excelente adaptación a la zona en la cual se trabajó la presente investigación. Estos resultados concuerdan con lo reportado por González et al. (2014), quienes señalaron que $Q$. humboldtii se adaptó muy bien en el departamento de Nariño, específicamente en los municipios de Ipiales, Funes y Pasto. De igual forma, Castillo y Calderón (2017) y Díaz et al. (2017), reconocieron esta 
especie como nativa del trópico alto, con potencial para contribuir a la conservación de la biodiversidad en sistemas productivos, y con ello, al desarrollo sostenible de los mismos.

La mortalidad y baja calidad de plantas evidenciada en la especie C. montana, se relaciona con la susceptibilidad de esta a suelos encharcados (Trujillo, 2013), ya que en la zona donde se estableció la especie prevalecen canales de agua que pueden generar encharcamientos, condición que influyó en el porcentaje de mortalidad. Debido a lo anterior, no sería propicio establecer esta especie en zonas con potencial de inundación o humedad elevada en el suelo.

En estudios desarrollados por Díaz (2007) y Trujillo y Vargas (2008), observaron que S. pyramidalis presentó tasas de crecimiento negativas y afectación en sobrevivencia, debido a la intolerancia a heladas y susceptibilidad a herbívoria. Sin embargo, en el presente estudio no se observaron afectaciones, por lo que se cataloga a $S$. pyramidalis como una de las especies de mejor adaptación en las condiciones de 2750 a 2800 msnm en trópico de altura de Nariño.

Aunque en A. acuminata no hubo afectación significativa en calidad de plantas $(\mathrm{p}<0,05)$, fue notable en las tres elevaciones evaluadas la quemadura de sus hojas, relacionada con factores climáticos extremos, como heladas. Resultados similares observaron Flores y Umaña (2006), en la evaluación de adaptación de acacia negra (Acacia decurrens), acacia japonesa (Acacia melanoxylon) y aliso (Alnus acuminata) en el trópico alto colombiano. Sin embargo, la especie A. acuminata se reporta como de uso frecuente en sistemas silvopastoriles tradicionales y potenciales para la agroforestería en el trópico alto colombiano (Murgueitio et al., 2011; Muñoz et al., 2013). Se debe considerar entonces, la asociación con otras especies de porte alto como roble ( $Q$. humboldtii) y caucho ( $F$. andicola), para reducir las afectaciones de las heladas; teniendo en cuenta estas dos especies como barreras protectoras durante las fases de establecimiento, crecimiento y desarrollo de otras especies.

A partir de lo reportado anteriormente, en cuanto a variables de calidad de plántulas, entre las especies con mejor calidad estuvieron: $Q$. humboldtii, S. pyramidalis y M. pubescens, las cuales se reconocen como nativas del trópico alto, por lo cual poseen la ventaja de desarrollarse bien y en menor tiempo (Laughlin, 2014). En estudios realizados sobre cercas vivas, Harvey et al. (2005) encontraron una amplia biodiversidad de especies donde la mayoría fueron nativas, lo cual demostró la importancia de estas cercas vivas en la generación de bienes y servicios ambientales, entre ellos, conservación de la biodiversidad, protección de fuentes hídricas, fijación de carbono, provisión de alimento para el ganado y aprovechamiento de productos forestales no maderables.

\section{Conclusión}

Se evidenció variación asociada a la altitud, principalmente para las variables morfométricas altura y diámetro; en donde las especies Ficus andicola a $2750 \mathrm{msnm}$ en estrato alto y Alnus acuminata y Smallanthus pyramidalis a $2800 \mathrm{msnm}$ en estrato medio, mostraron el mayor crecimiento inicial. Las especies Quercus humboldtii a 2770 y $2750 \mathrm{msnm}$, Ficus andicola a $2750 \mathrm{msnm}$, Smallanthus pyramidalis a $2800 \mathrm{msnm}$ y Morella pubescens (especies nativas) a $2800 \mathrm{msnm}$, sobresalieron en estrato alto, medio y bajo respectivamente, por su buena calidad de plantas en cerca viva multiestrato para protección de fuente hídrica en trópico altoandino nariñense. En esta investigación, las especies estudiadas evidenciaron potencial para ser establecidas en arreglos silvopastoriles de manera asociada, mediante diferentes estratos. Se podría aprovechar sus múltiples beneficios, potencializar su uso y así tratar de mejorar la eficiencia productiva de la ganadería bovina en trópico alto, sin efectos negativos sobre el ambiente. 


\section{Agradecimientos}

Los autores agradecen a la Corporación Colombiana de Investigación Agropecuaria (AGROSAVIA) donde se desarrolló el proyecto "Mejoramiento de la oferta forrajera, optimización de sistemas de alimentación y aseguramiento de la calidad e inocuidad de leche en el trópico alto del departamento de Nariño", financiado por el Sistema General de Regalías (SGR).

\section{Literatura citada}

Apráez, E., A. Gálvez, y C. Jojoa. 2014. Valoración nutricional y emisión de gases de algunos recursos forrajeros del trópico de altura. Rev. Cienc. Agric. 31:122-134. doi:10.22267/rcia.143102.36

Ávila, L., y O. Vargas. 2009. Formación de núcleos de restauración de Lupinus bogotensis dentro de claros en plantaciones de Pinus patula y Cupressus lusitánica. En: O. Vargas et al., editores, Restauración ecológica en zonas invadidas por retamo espinoso y plantaciones forestales de especies exóticas. Universidad Nacional de Colombia, Bogotá, COL. p. $234-306$.

Bare, M., and M. Ashton. 2015. Growth of native tree species planted in montane reforestation projects in the Colombian and Ecuadorian Andes differs among site and species. New For. 47:333-355. doi:10.1007/s11056-015-9519-z

Blanco, G., D. Chamorro, y L. Arreaza. 2005. Predicción de la respuesta productiva en bovinos lecheros suplementados con ensilaje de Sambucus peruviana, Acacia decurrens y Avena sativa usando el modelo Cornell Net Carbohydrate and Protein System (CNPS). Rev. CORPOICA 6(2):86-90. doi:10.21930/rcta.vol6_num2_art:53

Calle, Z., E. Murgueitio, y J. Chará. 2012. Integración de las actividades forestales con la ganadería extensiva sostenible y la restauración del paisaje. Unasylva 63(1):31-38. FAO, Roma, ITA. http://www.fao.org/docrep/017/i2890s/i2890s06.pdf (consultado 1 oct. 2018).

Cárdenas, C., C. Rocha, y J. Mora. 2011. Productividad y preferencia de forraje de vacas lecheras pastoreando un sistema silvopastoril intensivo de la zona alto Andina de Roncesvalles, Tolima. Rev. Col. Cienc. Anim. 4:29-35.

Cardona, W., P. Chacón, y G. Kattan. 2007. Avispas no polinizadoras asociadas a Ficus andicola (Moraceae) en la Cordillera Central de Colombia. Rev. Colom. Entomol. 33:165-170.

Castillo, Y., y J. Calderón. 2017. Plantas usadas por aves en paisajes cafeteros de Nariño, Colombia. Rev. Cienc. Agr. 34(2):318. doi: $10.22267 /$ rcia. 173402.68

Clavero, T., y J. Suárez. 2006. Limitaciones en la adopción de los sistemas silvopastoriles en México. Pastos y Forrajes 29(3):16.

DeFries, R., and C. Rosenzweig. 2010. Toward a whole-landscape approach for sustainable land use in the tropics. Nat. Acad. Sci. 107:19627-19632. doi:10.1073/pnas.1011163107

Delgado, I., J. Daza, H. Leonel, C. Luna, y A. Forero. 2016. Cuantificación de carbono radical Morella pubescens (Humb. \& Bonpl. ex Willd.) Wilbur en dos agroecosistemas (Nariño, Colombia). Colomb. For. 19(2):85-93. doi:10.14483/udistrital. jour.colomb.for.2016.2.a06

DNP (Departamento Nacional de Planeación). 2014. Política y estrategias para el desarrollo agropecuario del departamento de Nariño. Documento Conpes 3811. 2014. DNP, Bogotá, COL. https://colaboracion.dnp.gov.co/CDT/Conpes/ Econ\%C3\%B3micos/3811.pdf (consultado 29 set. 2018).

Díaz, A. 2007. Patrones de respuesta a heladas en arboles altoandinos sembrados en potreros y borde ripario potrerizado. En: O. Vargas, editor, Restauración ecológica del bosque Altoandino, Estudios diagnósticos y experimentales en los alrededores 
del Embalse de Chisacá. Universidad Nacional de Colombia, Acueducto de Bogotá, Jardín Botánico, y Secretaría Distrital de Ambiente, Bogotá, COL. p. 425-444.

Díaz, M., Y. Sepúlveda, y F. Moreno. 2017. Desarrollo inicial del roble andino en respuesta al manejo en vivero y durante la plantación. Colomb. For. 17:118-130. doi:10.14483/udistrital.jour.colomb.for.2017.2.a02

Estupiñán, L., y L.M. Cabrera. 2008. Los alrededores todo un ambiente en la U.D.C.A. Rev. U.D.C.A. Actual. Divulg. Cient. 11(2):3-9.

Fernández, J., y M. Hernández. 2007. Catálogo de la flora vascular de la cuenca alta del río Subachoque (Cundinamarca, Colombia). Caldasia 29:73-104.

Flebes, G., y T. Ruiz. 2008. Evaluación de especies arbóreas para sistemas silvopastoriles. Avan. Invest. Agropecu. 12(1):4-27.

Flores, L., y J. Umaña. 2006. Evaluación de la adaptación, comportamiento y efecto en la pradera de la acacia negra (Acacia decurrens), acacia japonesa (Acacia melanoxylon), y aliso (Alnus acuminata), como cerca viva en un sistema de producción de ganado de leche en el trópico alto colombiano. Tesis de grado. Universidad de la Salle, Bogotá D.C., COL.

García, G. 2009. Activación de la sucesión vegetal de especies promisorias parcela experimental permanente vereda pueblo viejo parte alta Facatativá (Colombia). Rev. Bras. Agroecol. 4:4021-4022.

González, C., A. Jarvis, and J. Palacio. 2014. Biogeography of the Colombian oak, Quercus humboldtii Bonpl: geographical distribution and their climatic adaptation. CIAT, Museo de Historia Natural, and Universidad del Cauca, Popayán, COL.

González, D., R. Poma, M. Ordoñez, y N. Aguirre. 2010. Crecimiento inicial de Tabebuia chrysantha y Cedrela montana con fines de rehabilitación de áreas abandonadas en el trópico húmedo en el Trópico Húmedo ecuatoriano. Ecol. For. 1(1):7380 .

Grajales, B., M. Botero, y J. Ramírez. 2015. Características, manejo, usos y beneficios del saúco (Sambucus nigra L.) con énfasis en su implementación en sistemas silvopastoriles del Trópico Alto. RIAA 6(1):155-168. doi:10.22490/21456453.1271

Gutiérrez, N. 2012. Estado de la vegetación en núcleos de restauración utilizados para el restablecimiento del bosque altoandino del Parque Forestal Embalse del Neusa, Cundinamarca, Colombia. Trabajo de grado. Pontificia Universidad Javeriana, Bogotá D.C., COL.

Harvey, C., F. Alpízar, M. Chacón, and R. Madrigal. 2005. Assessing linkages between agriculture and biodiversity in Central America: Historical overview and future perspectives. The Nature Conservancy (TNC), San José, CRI. doi:10.13140/ RG.2.1.2898.9524

Holdridge, L. 2000. Ecología basada en zonas de vida. 5a ed. IICA, San José, CRI.

Laughlin, D. 2014. Applying trait-based models to achieve functional targets for theory driven ecological restoration. Ecol. Lett. 17:771-784. doi:10.1111/ele.12288

León, O. 2007. Experimentos de restauración ecológica en plantaciones de Pinus patula. En: O. Vargas, editor, Restauración ecológica del bosque Altoandino, Estudios diagnósticos y experimentales en los alrededores del embalse de Chisacá (Localidad de Usme, Bogotá D.C). Universidad Nacional de Colombia, Acueducto de Bogotá, Jardín Botánico, y Secretaría Distrital de Ambiente, Bogotá, COL. p. 296-335.

Libreros, H. 2015. Sistemas silvopastoriles: opción para la mitigación y adecuación al cambio climático en bosque seco tropical. Rev. Semillas 57-58:62-67.

Luna, C. 2011. Laurel de cera (Morella pubescens), especie promisoria de usos múltiples empleada en agroforestería. Agrofor. Neotrop. 1:1. 
Machado, N., M. Prioli, A. Gatti, and V. Mendes. 2006. Temperature effects on seed germination in races of common beans (Phaseolus vulgaris L.). Acta Sci. Agron. 28:155-164.

Mahecha, G., A. Ovalle, D. Carmelo, A. Rozo, y D. Barrero. 2004. Vegetación del territorio CAR: 450 especies de sus llanuras y montañas. Corporación Autónoma Regional de Cundinamarca, Bogotá, COL.

Medina, M., H. Orozco, y M. Díez. 2008. Establecimiento de un sistema silvopastoril mediante las especies Alnus acuminata H.B.K. y Acacia decurrens Willd y respuesta al empleo de organismos rizosféricos en San Pedro (Antioquia). Livest. Res. Rural Dev. 20(1):7. http://www.lrrd.org/lrrd20/1/medi20007.htm (consultado 7 set. 2018).

Mendiburu, F. 2017. Agricolae: Statistical procedures for agricultural research. R package version 1.2-8. R Foundation for Statistical Computing, Vienna, AUS. https://cran.r-project.org/web/packages/agricolae/index.html (consultado 10 set. 2018).

Millán, H., y F.W. Moreno. 2005. Evaluación de adaptación al establecimiento en arbóreas multipropósito para sistemas ganaderos sostenibles en la Sabana de Bogotá. Tesis de grado. Universidad Nacional de Colombia, Bogotá, COL.

Molina, M., M. Medina, y L. Mahecha. 2008. Microorganismos y micronutrientes en el crecimiento y desarrollo del Aliso (Alnus acuminata H.B.K.) en un sistema silvopastoril alto andino. Livest. Res. Rural Dev. 20(4):54. http://www.lrrd.org/lrrd20/4/ moli20054.htm (consultado 7 set. 2018).

Montagnini, F. 2015. Función de los sistemas agroforestales en la adaptación y mitigación del cambio climático. En: F. Montagnini et al., editores, Sistemas agroforestales funciones productivas, socioeconómicas y ambientales. Informe Técnico 402. CATIE, Turrialba, CRI. p. 269-297.

Muñoz, D., D. Calvache, y J. Yela. 2013. Especies forestales con potencial agroforestal para las zonas altas en el departamento de Nariño. Rev. Cienc. Agric. 29(1):38-53.

Muñoz, D., J. Navia, y J. Solarte. 2018. El conocimiento local en los sitemas silvopastoriles tradicionales: Experiencias de investigación en la región andina. Editorial Universidad de Nariño, Nariño, COL.

Murgueitio, E., F. Uribe, A. Calle, and B. Solorio. 2011. Native trees and shrubs for the productive rehabilitation of tropical cattle ranching lands. For. Ecol. Manag. 261:1654-1663. doi:10.1016/j.foreco.2010.09.027

Murgueitio, E., M. Xóchitl, Z. Calle, J. Chará, R. Barahona, y C. Molina. 2015. Productividad de sistemas silvopastoriles intensivos en América Latina. En: F. Montagnini et al., editores, Sistemas agroforestales funciones productivas, socioeconómicas y ambientales. Informe Técnico 402. CATIE, Turrialba, CRI. p. 59-104.

Núñez-Florez, R., U. Pérez-Gómez, and F. Fernández-Méndez. 2019. Functional diversity criteria for selecting urban trees. Urban For. Urban Greening 38:251-266. doi:10.1016/j.ufug.2019.01.005

Orwa, C., A. Mutua, R. Kindt, R. Jamnadass, and A. Simons. 2009. Agroforestree database: A tree species reference and selection guide version 4.0. World Agroforestry Centre, Nairobi, KEN.

Pacheco, E., y A.S. Quisbert. 2016. Modelos de aprovechamiento sostenible del Aliso (Alnus acuminata Kunth) en zona de ladera de bosque de niebla. J. Selva Andina Biosph. 4(1):24-38.

Parmesan, C. 2006. Ecological and evolutionary responses to recent climate change. Ann. Rev. Ecol. Evol. Syst. 37:637-669. doi:10.1146/annurev.ecolsys.37.091305.110100

Pausas, J. 2010. Fuego y evolución en el mediterráneo. Invest. Cienc. 407:56-63.

Rivas, D. 2005. ¿Por qué el desmoche perjudica a los árboles? Sociedad Internacional de Arboricultura (ISA). Daniel Rivas, MEX. http://www.rivasdaniel.com/Articulos/Desmoche.pdf (consultado 4 dic. 2018). 
Sánchez, L., G. Amado, P. Criollo, T. Carvajal, J. Roa, A. Cuesta, A. Conde, A. Umaña, L. Bernal, y L. Barreto. 2010. El Saúco (Sambucus nigra L) como alternativa silvopastoril en el manejo sostenible de praderas en el trópico alto colombiano. CORPOICA, Medellín, COL.

Sánchez, S., y O. Murillo. 2004. Desarrollo de un método para controlar la calidad de producción de plántulas en viveros forestales: estudio de caso con ciprés (Cupressus lusitánica). Agron. Costarricense 28(2):95-106.

Sierra, M., y A. Amarillo. 2017. Características socioecológicas de la diversidad de plantas en huertos domésticos en la ciudad de Bogotá, Colombia. Urban For. Urban Greening 28:54-62. doi:10.1016/j.ufug.2017.09.015

Tang, C.Q., X. Hou, T. Xia, C. Duan, and D. Fu. 2007. Man-made versus natural forests in Mid-Yunnan, southwestern China. Mt. Res. Dev. 27:242-249. doi:10.1659/mrd.0732

Tovar, G. 2013. Aproximación a la silvicultura urbana en Colombia. Bitácora 22:119-136.

Trujillo, E. 2013. Los árboles: adaptación, producción y silvicultura, madera, usos. En: E. Trujillo, editor, Guía de reforestación: ilustración, aumentada y corregida. 3a ed. El Semillero, Bogotá, COL.

Trujillo, L., y O. Vargas. 2008. Crecimiento y supervivencia de especies nativas en bordes de avance. En: O. Vargas, editor, Estrategias para la restauración ecológica del bosque altoandino: El caso de la Reserva Forestal Municipal de Cogua, Cundinamarca. 2a ed. Universidad Nacional de Colombia, Bogotá, COL. p. 307-372.

UMAIC (Unidad de Manejo y Análisis de Información Colombia). 2017. Briefing departamental Nariño 2017. UMAIC, COL. https://umaic.org/briefings/2017/Narino_2017.pdf (accessed Oct. 23, 2018).

Uribe, F., y A. Zuluaga. 2011. Manejo de agua en fincas ganaderas. En: F. Uribe, et al., editores, Buenas prácticas ganaderas. Manual 3. Proyecto ganadería colombiana sostenible. GEF, Banco Mundial, FEDEGAN, CIPAV, Fondo Acción, y TNC, Bogotá, COL. p. 40-45.

Wickham, H. 2016. Ggplot2: Create elegant data visualisations using the grammar of graphics. R package version 3.1.0. R Foundation for Statistical Computing, Vienna, AUS. https://cran.r-project.org/web/packages/ggplot2/index.html (accessed Sep. 10, 2018).

Zepeda, R., M. Velasco, J. Nahed, A. Hernández, y J. Martínez. 2016. Adopción de sistemas silvopastoriles y contexto sociocultural de los productores: apoyos y limitantes. Rev. Mex. Cienc. Pecu. 7:471-488. doi:10.22319/rmcp.v7i4.4282 